\title{
Perfil clínico e epidemiológico de pessoas com diagnóstico de tuberculose em um município do interior paulista
}

\author{
Clinical and epidemiological profile of a patient with tuberculosis treated in a city in the \\ interior of São Paulo
}
Perfil clínico-epidemiológico de personas diagnosticadas con tuberculosis en una ciudad del interior de São Paulo

Israel Lucas da Silva Maza ${ }^{1 *}$, Pedro Augusto Bossonario² ${ }^{2}$ Nanci Michele Saita ${ }^{2}$, Aline Aparecida Monroe $^{2}$, Lívia Maria Lopes-Gazaffi ${ }^{1}$.

\section{RESUMO}

Objetivo: Descrever o perfil clínico e epidemiológico de pessoas com diagnóstico de tuberculose atendidas na rede pública e notificadas em um município do interior paulista. Métodos: Estudo descritivo, quantitativo, cujos dados foram coletados no Sistema TBWEB e em planilhas do serviço de vigilância epidemiológica em um município do interior de São Paulo. Foram incluídos todos os casos de tuberculose notificados entre 2013 e 2018. Utilizou-se variáveis sociodemográficas, clínicas, informações de diagnóstico e tratamento; analisadas pelo programa Statistic 12.0 por meio de medidas de frequência absoluta e relativa para as variáveis categóricas. Resultados: Foram notificados 375 casos com idade média de 43,1 anos, sendo a maioria do sexo masculino $(72,8 \%)$, cor branca $(53,6 \%)$ e escolaridade de 4 a 11 anos $(67,7 \%)$. Identificouse que $84,8 \%$ apresentaram a forma clínica pulmonar e $82,9 \%$ dos casos realizaram baciloscopia de escarro. Em relação a suspeita de tuberculose, $71,7 \%$ dos indivíduos realizaram raio-X. Quanto à sorologia para HIV, foram realizados testes em $95,1 \%$ dos casos, dos quais $10 \%$ obtiveram resultado positivo. Conclusão: A investigação possibilitou compreender as características clínicas e epidemiológicas da tuberculose, além de reflexões acerca da organização da rede de atenção em saúde diante dos casos de tuberculose.

Palavras-chave: Tuberculose, Doenças transmissíveis, Saúde pública, Perfil epidemiológico.

\begin{abstract}
Objective: To describe the clinical and epidemiological profile of people diagnosed with tuberculosis treated in the public healthcare and notified in a city in the interior of São Paulo. Methods: Descriptive, quantitative study, whose data were collected in the TBWEB system and in spreadsheets from the epidemiological surveillance service in the study city. All tuberculosis cases notified between 2013 and 2018 were included. Sociodemographic and clinical variables, diagnosis and treatment information were used; analyzed by the Statistic 12.0 program through measures of absolute and relative frequency for categorical variables. Results: A total of 375 cases were reported, with a mean age of 43.1 years, most of them male $(72.8 \%)$, white (53.6\%) and with 4 to 11 years of schooling (67.7\%). It was identified that $84.8 \%$ had the clinical pulmonary form and $82.9 \%$ of the cases underwent sputum smear microscopy. Regarding suspected tuberculosis, $71.7 \%$ of individuals underwent X-rays. As for HIV serology, tests were performed in $95.1 \%$ of cases, of which $10 \%$ were positive. Conclusion: The investigation made it possible to understand the clinical and epidemiological characteristics of tuberculosis, in addition to enabling reflection on the organization of the health care network in the face of tuberculosis cases.
\end{abstract}

Keywords: Tuberculosis, Communicable diseases, Public health, Health profile.

\section{RESUMEN}

Objetivo: Describir el perfil clínico-epidemiológico de las personas diagnosticadas con tuberculosis atendidas en la red pública y notificadas en una ciudad del interior de São Paulo. Métodos: Estudio descriptivo, cuantitativo, cuyos datos fueron recolectados en el Sistema TBWEB y en planillas del servicio de vigilancia

${ }^{1}$ Centro Universitário Municipal de Franca (UNI-FACEF), Franca - SP. *E-mail: israelucas2008@gmail.com

2 Escola de Enfermagem de Ribeirão Preto (USP), Ribeirão Preto - SP

Fonte de apoio: Programa de Iniciação Científica Uni-FACEF

SUBMETIDO EM: 10/2021

ACEITO EM: 10/2021

PUBLICADO EM: 10/2021 
epidemiológica en la ciudad de estudio. Se incluyeron los casos de tuberculosis notificados entre 2013-2018. Se utilizaron variables sociodemográficas, clínicas, diagnóstico y tratamiento; analizados por el programa Statistic 12.0 a través de medidas de frecuencia absoluta y relativa para variables categóricas. Resultados: Se reportaron un total de 375 casos, con edad media de 43,1 años, la mayoría varones (72,8\%), blancos $(53,6 \%)$, con 4 a 11 años de escolaridad $(67,7 \%)$. Se identificó que $84,8 \%$ presentaba la forma clínica pulmonar y $82,9 \%$ de los casos se sometió a microscopía de baciloscopia de esputo. La sospecha de tuberculosis, en $71,7 \%$ de los individuos se sometieron a radiografías. En cuanto a la serología del VIH, se realizaron pruebas en $95,1 \%$ de los casos, de los cuales $10 \%$ fueron positivos. Conclusión: La investigación permitió comprender las características clínicas / epidemiológicas de la tuberculosis, además de posibilitar la reflexión sobre la organización de la red asistencial ante los casos de tuberculosis.

Palabras clave: Tuberculosis, Enfermedades transmisibles, Salud pública, Perfil de salud.

\section{INTRODUÇÃO}

Estima-se que, no mundo, 10 milhões de pessoas adoeceram por Tuberculose (TB) em 2019, sendo que 1,2 milhão de adultos e crianças foram a óbito e 208 mil viviam com o Vírus da Imunodeficiência Humana (HIV). O Brasil é um dos países com maior número de casos, estando entre os 30 países com alta carga epidemiológica de TB e de coinfecção TB-HIV (WORLD HEALTH ORGANIZATION, 2020). Desde 2003, considera-se como prioritária a doença na agenda política do Ministério da Saúde, havendo aproximadamente, 69 mil casos novos e 4.500 óbitos a cada ano, no qual a causa básica é a TB (MINISTÉRIO DA SAÚDE, 2017).

No ano de 2020, no estado de São Paulo, foram registrados 15.823 casos novos de TB, com uma incidência de 34,4 casos a cada 100 mil pessoas, além disso, houve 901 óbitos causados pela doença, sendo que o perfil dos indivíduos acometidos era, na maioria, homens e na faixa etária dos 15 aos 59 anos. No mesmo ano, o estado registrou 1.111 indivíduos coinfectados com TB-HIV, representando 7\% dos casos (MINISTÉRIO DA SAÚDE, 2021).

No Brasil, os casos de TB são notificados, principalmente, nas regiões de periferia, em áreas de aglomeração ou locais privados de ventilação e iluminação (GIERGOWICZ FB e MAHMUD IC, 2020). A alimentação e os hábitos de vida também se tornam um fator determinante para infecção, uma vez que se relaciona à ingestão de álcool, consumo de tabaco e outras drogas (SANTOS JUNIOR CJ, et al., 2019; GIERGOWICZ FB e MAHMUD IC, 2020). Diante das vulnerabilidades expostas, também merece destaque a coinfecção TB-HIV, uma vez que o indivíduo pode ter o quadro de saúde agravado e levar a um desfecho desfavorável em situações nas quais não exista o diagnóstico e tratamento oportuno (CAVALIN RF, 2018).

Além disso, a população brasileira enfrenta barreiras no acesso e na busca aos serviços de saúde, pois, muitas vezes, estes fatores estão associados a situações socioeconômicas, à falta de conhecimento quanto à gravidade da doença, à distância e ao acesso às unidades de saúde entre outros fatores, como a baixa efetividade do diagnóstico e a baixa percepção dos profissionais que atuam nesses serviços quando lidam com pacientes com TB, gerando várias idas aos serviços, além de refletir na subnotificação dos casos e na dificuldade em traçar o perfil sociodemográfico e clínico, impossibilitando o planejamento e avaliação de programas direcionados ao controle da doença (LEITE RC, 2016; PONCE MAZ, et al., 2016).

O diagnóstico da tuberculose pode ser clínico, bacteriológico, radiológico, histopatológico e por técnicas biomoleculares, sendo que se preconiza a solicitação da baciloscopia quando existe a suspeita da doença, possibilitando o diagnóstico precoce, a notificação dos casos e os desfechos favoráveis, apesar das limitações do teste (MALACARNE J, et al., 2019). A busca ativa, realizada pela Atenção Primária à Saúde (APS), contribui para a detecção de casos, constituindo-se como a principal forma de controle da TB (MINISTÉRIO DA SAÚDE, 2019). Entretanto, a porcentagem de casos diagnosticados no contexto hospitalar e em serviços de pronto atendimento são elevadas no Brasil, revelando a fragilidade na assistência à saúde e o diagnóstico tardio (RANZANI OT, et al., 2018).

As estratégias para controle da TB no Sistema Único de Saúde (SUS) elencam ações informativas, preventivas e assistenciais, como imunização, identificação de sintomáticos respiratórios, testagem para o 
HIV, consultas de acompanhamento e oferta do Tratamento Diretamente Observado (TDO), visando minimizar as taxas de abandono do tratamento e, consequentemente, casos resistentes. $\mathrm{A}$ adesão ao tratamento ou abandono é influenciada por questões de vulnerabilidade social, como a pobreza, o uso de drogas e as pessoas sem teto (JUNGES JR, et al., 2020).

A elaboração deste estudo pretendeu descrever o perfil de adoecimento das pessoas notificadas e diagnosticadas com tuberculose e compreender as características dos indivíduos em adoecimento pela TB, bem como as fragilidades e demandas que vulnerabilizam o sujeito, contribuindo para o pensamento e planejamento de medidas de prevenção e ampliação do diagnóstico oportuno, a fim de diminuir e controlar o percurso da doença.

\section{MÉTODOS}

Trata-se de um estudo descritivo, epidemiológico e retrospectivo realizado com base nos dados da Secretaria de Saúde de um município do interior de São Paulo, que recebe informações geradas nos serviços de saúde do município, o qual possui 355 mil habitantes (INSTITUTO BRASILEIRO DE GEOGRAFIA E ESTATÍSTICA, 2020).

Os serviços que ofertam ações para o controle da TB no município estudado integram uma rede de saúde composta por 14 Unidades Básicas de Saúde (UBS) e cinco Estratégias de Saúde da Família (ESF). Quanto ao atendimento de urgência, o município dispõe de duas Unidades de Pronto Atendimento (UPA), um Pronto Socorro Municipal para adultos, além de outros serviços voltados para população infantil e demais comorbidades (SECRETARIA DE SAÚDE, 2021)

A população deste estudo constituiu-se por sujeitos que tiveram diagnóstico de TB com notificação no município estudado no período de 2013 a 2018 e que fizeram acompanhamento no Ambulatório de Controle da Tuberculose municipal. Foram adotados como critérios de inclusão pessoas com idade igual ou superior a 18 anos; casos com diagnóstico confirmatório de TB (casos novos, recidiva e retratamento por abandono) e residentes da cidade estudada. Foram excluídos aqueles notificados por outros municípios, mesmo que estivessem em acompanhamento no cenário de estudo.

A coleta de dados foi realizada por meio de fontes secundárias de informação obtidas pelo TBWEB após solicitação de um banco de dados à Secretaria de Saúde municipal, no qual foi possível consultar informações sociodemográficas; dados gerais sobre o perfil clínico dos casos diagnosticados com TB e o controle da doença estudada. Em seguida, os dados foram transferidos para uma planilha do Excel e realizados os cálculos estatísticos.

A análise dos dados se deu por meio do programa Statistic 7.0, utilizando-se técnicas de estatística descritiva: medidas de frequência absoluta e frequência relativa para as variáveis categóricas. Para as variáveis numéricas, foram calculadas medidas de posição (média e mediana) e de variabilidade (desvio padrão).

O presente estudo foi aprovado pelo Comitê de Ética em Pesquisa do Centro Universitário Municipal de Franca (Uni-FACEF) sob protocolo 08973018.5.0000.5384 após anuência da Secretaria Municipal de Saúde. Mediante ao período de realização do estudo e a lei vigente da época, houve dispensação da aplicação do Termo de Consentimento Livre Esclarecido (TCLE) por se tratar de um estudo com coleta de dados secundários.

\section{RESULTADOS}

Foram notificados no período compreendido entre janeiro de 2013 e dezembro de 2018, 375 casos de Tuberculose no município estudado, dos quais 327 foram considerados casos novos. O maior número de casos observados foi no ano de 2017 , com $20,2 \%$ do total e coeficiente de incidência de casos novos de $0,1 \%$, tendo como base o último censo do município (IBGE, 2020). A distribuição dos casos incluídos para análise está descrita, de acordo com os anos, no Gráfico 1. 
Gráfico 1 - Distribuição da incidência de pessoas com tuberculose no município de Franca-SP, 2013 a 2018.

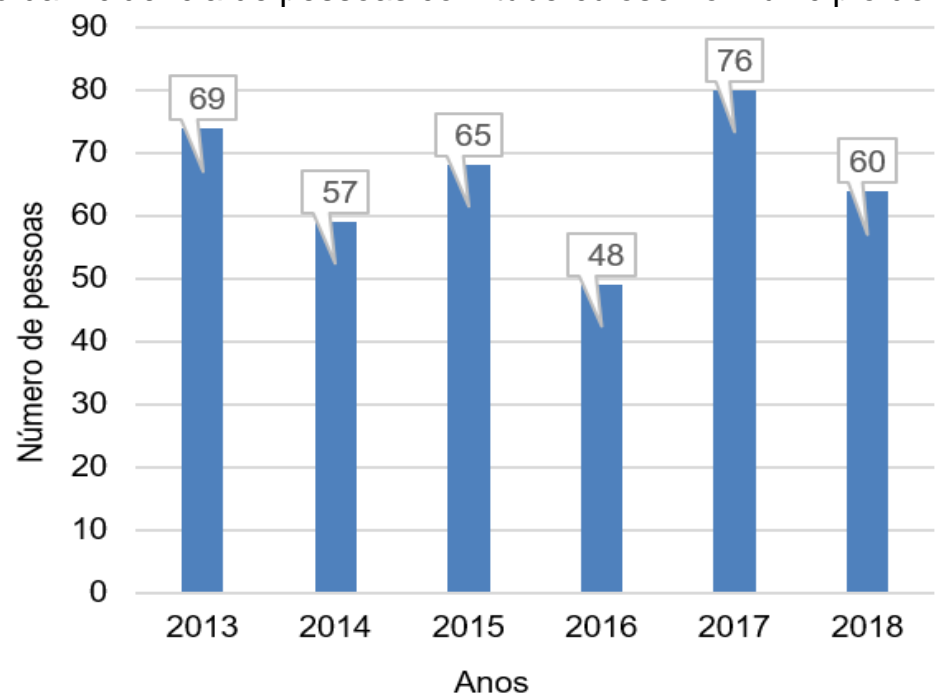

Fonte: Maza ILS, et al., 2021; dados extraídos do Sistema de Informação TBWEB, Secretaria de Saúde de Franca, SP.

Entre os participantes, a média de idade geral foi de $43,1( \pm 16,6)$ anos, com predominância de faixa etária de 18 a 39 anos, sexo masculino (72,8\%), raça/cor branca $(53,6 \%)$, prevalência de quatro a sete anos de estudo (36\%) e com ocupação não especificadas (48\%). Aposentados e desempregados representam 39,2\% do total (Tabela 1).

Tabela 1 - Distribuição de frequência das características sociodemográficas de pessoas com tuberculose, Franca - SP, 2013 a 2018.

\begin{tabular}{|c|c|c|}
\hline Características Sociodemográficas & $\mathbf{n}$ & $\%$ \\
\hline \multicolumn{3}{|l|}{ Sexo } \\
\hline Masculino & 273 & 72,8 \\
\hline Feminino & 102 & 27,2 \\
\hline \multicolumn{3}{|l|}{ Faixa etária } \\
\hline $18-39$ & 189 & 50,4 \\
\hline $40-59$ & 117 & 31,2 \\
\hline$>60$ & 69 & 18,4 \\
\hline \multicolumn{3}{|l|}{ Raça/Cor } \\
\hline Branco & 201 & 53,6 \\
\hline Pardo & 81 & 21,6 \\
\hline Preto & 53 & 14,1 \\
\hline Não informado & 40 & 10,7 \\
\hline \multicolumn{3}{|l|}{ Escolaridade } \\
\hline Sem escolaridade & 11 & 3,0 \\
\hline De 1 a 3 anos & 40 & 10,6 \\
\hline De 4 a 7 anos & 135 & 36,0 \\
\hline De 8 a 11 anos & 119 & 31,7 \\
\hline De 12 a 14 anos & 13 & 3,4 \\
\hline$>15$ anos & 14 & 3,9 \\
\hline Não informado & 43 & 11,4 \\
\hline \multicolumn{3}{|l|}{ Tipo de Ocupação } \\
\hline Desempregado & 95 & 25,3 \\
\hline Aposentado & 51 & 13,6 \\
\hline Não Informado & 19 & 5,0 \\
\hline Privado de liberdade & 14 & 3,8 \\
\hline Dona de casa & 9 & 2,4 \\
\hline Profissional de saúde & 6 & 1,7 \\
\hline Profissional de unidade prisional & 1 & 0,2 \\
\hline Outras & 180 & 48,0 \\
\hline
\end{tabular}

Fonte: Maza ILS, et al., 2021; dados extraídos do sistema de informação TB WEB, Secretaria de Saúde de Franca, SP. 
Quanto às comorbidades, $36,5 \%$ e $29,8 \%$ eram etilistas e tabagistas, respectivamente, além de $26,1 \%$ consumirem drogas ilícitas. Ainda, 5,6\% possuíam aids, 5,3\% Diabetes Mellitus e 21,3\% com menção de outros agravos à saúde (Tabela 2).

A forma clínica de TB pulmonar foi predominante, com $84,8 \%$ do total de indivíduos (tabela 2). Dos exames diagnósticos utilizados como critérios para confirmação de TB, a baciloscopia de escarro foi oferecida a 82,9\% dessa população, sendo positivo em $54,4 \%$ dos casos, seguido pela cultura de escarro, realizada com $48,8 \%$ das pessoas que obtiveram resultado positivo. O raio- $X$ foi avaliado em $71,7 \%$ dos casos, sendo que desses $45,3 \%$ foram considerados com suspeita de TB e 22,4\% suspeitos com cavidade. Em relação ao HIV, 95,1\% dos participantes realizaram testes sorológicos, sendo 10\% com resultado positivo (Tabela 2).

Tabela 2 - Distribuição de frequência das características clínico-epidemiológicas dos pacientes com tuberculose, Franca - SP, 2013 a 2018.

\begin{tabular}{|c|c|c|}
\hline Características clínico-epidemiológicas & $\mathbf{n}$ & $\%$ \\
\hline \multicolumn{3}{|l|}{ Comorbidades } \\
\hline Tabagismo & 137 & 36,5 \\
\hline Etilismo & 112 & 29,8 \\
\hline Uso de drogas ilícitas & 98 & 26,1 \\
\hline Aids & 21 & 5,6 \\
\hline Diabetes Mellitus & 20 & 5,3 \\
\hline Transtorno Mental & 3 & 0,8 \\
\hline Outras & 80 & 21,3 \\
\hline \multicolumn{3}{|l|}{ Forma Clínica } \\
\hline Pulmonar & 318 & 84,8 \\
\hline Extrapulmonar & 56 & 15,0 \\
\hline Pulmonar + Extra & 1 & 0,2 \\
\hline \multicolumn{3}{|l|}{ Baciloscopia de escarro } \\
\hline Positivo & 204 & 54,4 \\
\hline Negativo & 107 & 28,5 \\
\hline Não realizado & 64 & 17,1 \\
\hline \multicolumn{3}{|l|}{ Cultura de Escarro } \\
\hline Positivo & 183 & 48,8 \\
\hline Negativo & 69 & 18,4 \\
\hline Não realizado & 119 & 31,8 \\
\hline Não informado & 4 & 1,0 \\
\hline \multicolumn{3}{|l|}{ Raio-X } \\
\hline Suspeito de TB & 170 & 45,3 \\
\hline Suspeito com cavidade & 84 & 22,4 \\
\hline Normal & 12 & 3,2 \\
\hline Outra patologia & 3 & 0,8 \\
\hline Não realizado & 95 & 25,3 \\
\hline Não informado & 11 & 3,0 \\
\hline \multicolumn{3}{|l|}{ Sorologia para HIV } \\
\hline Negativo & 319 & 85,1 \\
\hline Positivo & 37 & 10,0 \\
\hline Não realizada & 13 & 3,4 \\
\hline Não informado & 6 & 1,5 \\
\hline
\end{tabular}

Fonte: Maza ILS, et al., 2021; dados extraídos do sistema de informação TBWEB, Secretaria de Saúde de Franca, SP.

Entre os 375 participantes, $87,2 \%$ eram casos novos. O diagnóstico foi obtido no ambulatório em $53 \%$ dos casos e em 15,4\% em unidades de urgência e emergência. Além disso, 24\% dos indivíduos receberam internação em um hospital filantrópico do município. Em relação ao tratamento diretamente observado (TDO), $96 \%$ realizaram a tomada da medicação nesta modalidade de tratamento. Quanto ao desfecho da doença, $69,6 \%$ obtiveram a cura, $10,6 \%$ abandonaram o tratamento e 2,9\% foram a óbito por TB (Tabela 3 ). 
Tabela 3 - Distribuição de frequência dos dados sobre o acompanhamento dos pacientes com tuberculose, Franca - SP, 2013 a 2018.

\begin{tabular}{|c|c|c|}
\hline Acompanhamento dos casos de TB & $\mathbf{n}$ & $\%$ \\
\hline \multicolumn{3}{|l|}{ Diagnóstico } \\
\hline Demanda ambulatorial & 199 & 53,0 \\
\hline Elucidação de diagnóstico de internação & 90 & 24,0 \\
\hline Urgência e emergência & 58 & 15,4 \\
\hline Investigação de contatos & 13 & 3,4 \\
\hline Busca ativa em instituição & 4 & 1,0 \\
\hline Busca ativa na comunidade & 1 & 0,2 \\
\hline Não informado & 10 & 3,0 \\
\hline \multicolumn{3}{|l|}{ Tratamento } \\
\hline Supervisionado & 360 & 96,0 \\
\hline Auto administrado & 4 & 1,0 \\
\hline Sem informação & 11 & 3,0 \\
\hline \multicolumn{3}{|l|}{ Tipo de caso } \\
\hline Novo & 327 & 87,2 \\
\hline Recidiva & 26 & 7,0 \\
\hline Retratamento por abandono & 22 & 5,8 \\
\hline \multicolumn{3}{|l|}{ Desfecho do tratamento } \\
\hline Cura & 261 & 69,6 \\
\hline Abandono & 40 & 10,6 \\
\hline Em tratamento ambulatorial & 19 & 5,0 \\
\hline Óbito Não TB & 18 & 4,8 \\
\hline Mudança de diagnóstico & 17 & 4,5 \\
\hline Óbito por TB & 10 & 2,9 \\
\hline Transferência & 7 & 2,0 \\
\hline Faltoso & 1 & 0,2 \\
\hline Falência/resistência & 1 & 0,2 \\
\hline Não informado & 1 & 0,2 \\
\hline
\end{tabular}

Fonte: Maza ILS, et al., 2021; dados extraídos do sistema de informação TBWEB, Secretaria de Saúde de Franca, SP.

\section{DISCUSSÃO}

Entre os anos de 2011 e 2016, foi observado uma constante tendência de queda no registro dos casos de TB no Brasil. Contudo, o coeficiente de incidência da doença aumentou entre os anos de 2017 e 2019, tendo esse último ano 37,4 casos por 100 mil habitantes. Não obstante, em 2020, houve uma diminuição no número de casos novos de TB, perfazendo um coeficiente de incidência de 31,6 / 100 mil habitantes, atribuindo-se essa queda à pandemia de COVID-19 (MINISTÉRIO DA SAÚDE, 2021).

Os casos de TB incluídos nesse estudo revelaram predominância do sexo masculino, sendo quase três vezes maior do que em relação ao sexo feminino. Este achado converge com a literatura, uma vez que uma pesquisa realizada no município de São Paulo apresentou a mesma proporção durante o ano de 2013, apesar da incidência anual entre os homens se manter estável durante a trajetória da doença (PINTO PFPS, et al., 2017).

A verificação desta ocorrência pode estar associada ao fato de homens possuírem dificuldades em procurarem os serviços de saúde para o cuidado consigo, devido questões culturais e sociais, demonstrando a importância e a possibilidade de ações de saúde realizadas pela APS junto de populações específicas, além da construção do conhecimento acerca dos modos de transmissão e prevenção à TB (TAVARES CM, et al., 2020).

Entre os achados, o maior número de indivíduos com TB esteve concentrado entre as idades de 20 e 39 anos para ambos os sexos, seguindo o perfil nacional, que abrange maior número de casos na faixa etária dos 15 aos 59 anos (MINISTÉRIO DA SAÚDE, 2018). As pessoas acometidas pela doença durante a fase jovem podem gerar impactos econômicos, pois, além do desequilíbrio financeiro familiar, existe o afastamento laboral do sujeito em fase economicamente produtiva (SANTOS JUNIOR CJ, et al., 2019). Compreender o 
perfil dos indivíduos acometidos pela TB é uma importante tarefa para que haja um direcionamento nas atividades de promoção em saúde e rastreamento de casos sintomáticos entre a população jovem, possibilitando, além de ações voltadas para temática, a oferta de testes sorológicos para HIV e o tratamento precoce, uma vez que há um aumento no número de pessoas jovens vivendo com HIV (MINISTÉRIO DA SAÚDE, 2020).

A variável raça/cor branca esteve presente em mais da metade dos casos estudados. Em contrapartida, verifica-se no cenário nacional uma predominância da cor preta e parda, atribuindo esse fato a maior vulnerabilidade desses grupos (MINISTÉRIO DA SAÚDE, 2021; TAVARES CM, et al., 2020). No presente município, há um maior número de pessoas brancas, seguidos de pardos e negros, evidenciando, também, a influência das regiões do Brasil nesse tipo de variável (IBGE, 2020).

A TB é uma doença relacionada diretamente com a pobreza, que pode ser determinada pelo baixo grau de instrução e desemprego (THOMÉ HR, et al., 2020). Nesse estudo, foi possível identificar que as características predominantes na casuística nacional estão relacionadas à baixa instrução e à ocupação das pessoas com TB, concordando com o perfil brasileiro (MINISTÉRIO DA SAÚDE, 2017). Observa-se predominância entre as pessoas com TB em relação à menor escolaridade, seguido de pessoas desempregadas, evidenciando uma condição que reflete em um conjunto de determinantes socioeconômicos precários, que aumentam a vulnerabilidade à TB e diminui a adesão ao tratamento (THOMÉ HR, et al. 2020; SILVA TO, et al., 2021). Assim, garantir o entendimento do indivíduo doente quanto ao uso das medicações, contribui para que as políticas públicas sejam alcançadas em relação à diminuição da TB e para desfechos favoráveis (COSTA AFA, et al., 2020)

No tocante das comorbidades e hábitos de vida associados à TB, a maior predominância está relacionada ao tabagismo, alcoolismo e outras drogas, respectivamente, nesse estudo. Entre os 22 países que sofrem com o alto impacto da doença, incluindo o Brasil, estima-se que mais de $20 \%$ da incidência de TB possa ser atribuída ao tabagismo ativo, além de existir evidências de associação entre o tabaco e a infecção por TB, proporcionando maiores números de recidiva e mortalidade (MINISTÉRIO DA SAÚDE, 2019). Atividades de promoção à saúde e o rastreio de indivíduos tabagistas podem contribuir para o controle da epidemia do tabaco, favorecendo a diminuição dos casos de TB existentes e a oferta de testes para o diagnóstico oportuno da TB em tabagistas sintomáticos (TONIN E, 2021).

A classificação e a forma clínica com maior número de casos no período de coleta de dados foi a TB pulmonar, seguido de um número reduzido de TB extrapulmonar. Esses achados corroboram com outros estudos que apresentaram a TB pulmonar como prevalente na população brasileira (TAVARES CM, et al., 2020; SILVA LT, et al., 2019; SANTOS JUNIOR CJ, et al., 2019). O Ministério da Saúde preconiza uma distribuição de $80 \%$ de casos pulmonares e $20 \%$ extrapulmonares na população geral e, apesar da doença ter a capacidade de acometer vários órgãos, a forma pulmonar continua a mais frequente e com maior valor epidemiológico em decorrência da sua alta transmissibilidade (MINISTÉRIO DA SAÚDE, 2019).

A baciloscopia de escarro foi o critério de confirmação mais presente no estudo, sendo um dos métodos mais comuns utilizados pela saúde pública para diagnosticar a doença, que, quando executado corretamente em todas as suas fases, permite detectar de $60 \%$ a $80 \%$ dos casos de TB pulmonar (MINISTÉRIO DA SAÚDE, 2019). Contudo, as limitações existentes nesse tipo de teste são observadas pela necessidade de uma grande quantidade de bacilos, o que possibilita interpretações de falsos negativos (FREITAS WMTM, et al., 2016).

O segundo maior critério observado para o diagnóstico de TB entre os indivíduos envolvidos nesse estudo foi a cultura de escarro, a qual possui elevada especificidade e sensibilidade (MINISTÉRIO DA SAÚDE, 2019). O exame radiológico sugestivo de TB foi possível também na maioria dos casos, sendo recomendado pelo Ministério da Saúde para todo pessoa com suspeita de TB pulmonar (MINISTÉRIO DA SAÚDE, 2019).

Foi identificado que das sorologias de HIV realizadas, a minoria apresentou teste positivo ou não realizou, embora 5,6\% das pessoas com TB já tinham o diagnóstico de aids. Apesar do baixo número de coinfecção TB/HIV, é recomendada a oferta de exames sorológicos, devido a contribuição observada no processo de diagnóstico oportuno do vírus (FREITAS WMTM, et al., 2016). A sorologia é um teste simples, disponível em 
unidades básicas de saúde, sendo preconizado para todos as pessoas portadoras de TB, pois o diagnóstico de HIV em pessoas com TB necessita da potencialização da eficácia do tratamento, visto que a presença do vírus repercute em graves implicações no controle da transmissão, como o óbito e outros desfechos desfavoráveis (FREITAS WMTM, et al., 2016).

A letalidade elevada de pessoas com TB e HIV pode estar relacionada a fatores como o diagnóstico tardio, adesão baixa à terapêutica medicamentosa e também ao desenvolvimento de resistência ao tratamento, sendo de grande importância a capacitação e sensibilização dos profissionais de saúde para uma abordagem integral das pessoas com TB (BASTOS SH, et al., 2020; COSTA AFA, et al., 2020).

É valido destacar que o vínculo e ações promotoras de saúde entre profissional e pessoa com TB, demonstram-se ferramentas necessárias para difundir informações, ampliar o incentivo às medidas de prevenção em saúde e responsabilização dos indivíduos, possibilitando o autocuidado e uma maior aproximação com pessoa doente (COSTA AFA, et al., 2020). Com isso, as características clínicas e epidemiológicas podem ser traçadas, constatando-se quais agravos de saúde são mais desfavoráveis para cura, como no caso das pessoas que vivem com HIV e tiveram o diagnóstico de TB, uma vez que esses indivíduos podem sofrer preconceitos e estigmas que os impeçam de expor a realidade que vivem (CAMPOY LT, et al., 2019)

Em relação ao local de diagnóstico da TB, observou-se maior proporção de casos nas demandas ambulatoriais da APS. Entretanto, quase metade dos casos foram identificados nos serviços de urgência e emergência e por elucidação do diagnóstico de internação hospitalar. O Ministério da Saúde, no Manual de Recomendações sobre a TB, destaca a preconização da busca ativa realizada pela APS, sendo esta uma das principais estratégias de controle para a TB (MINISTÉRIO DA SAÚDE, 2019). Ademais, o indivíduo doente pode aguardar um longo tempo para a procura de serviços de saúde desde o aparecimento dos primeiros sintomas quando a busca é realizada de forma passiva, uma vez que este modo se relaciona a autopercepção dos sinais e sintomas da TB, sendo um fator determinante para o atraso do diagnóstico oportuno e o agravo da patologia (DANTAS, et al, 2018).

A APS mantém-se como um dos serviços com menor procura ao início dos sintomas, justificada pela baixa credibilidade da população, burocracia para o atendimento, filas, demora para entrega dos exames e descontinuidade do cuidado, além das influências culturais que são medicocentradas e hospitalocêntricas, fomentada pela constituição hegemônica dos serviços de saúde (PONCE et al, 2016; TOMBERG et al, 2020). As dificuldades em centralizar o cuidado na atenção primária têm sido atribuídas ao baixo percentual na suspeição e solicitação de exames para diagnóstico, ao encaminhamento do usuário a outros serviços para realização de diagnóstico, entre outros. Essas características são indicativos de uma menor capacidade de resolução dos casos, endossando na população que as atenções especializadas são mais resolutivas do que a atenção básica (TOMBERG JO, et al., 2020).

O manejo da tuberculose no município é, em grande parte, realizado por meio do TDO, o qual possui como objetivo a observação da ingesta farmacológica para o tratamento da TB por um profissional da saúde do início até a cura (MINISTÉRIO DA SAÚDE, 2019). Embora a maioria dos casos terem sido considerados novos, as recidivas ou retratamento por abandono foram baixos neste estudo, demonstrando que, apesar do pequeno número de indivíduos que não aderiram ao tratamento, é importante o acompanhamento daqueles que abandonaram a terapêutica medicamentosa, pois uma das principais limitações para o efetivo controle da TB é a não adesão, que implica não só no custo do seguimento, como também na mortalidade e nas taxas de recidiva da doença, facilitando o desenvolvimento de bacilos mais resistentes (RÊGO RT, et al., 2018).

Entretanto, apesar dos abandonos verificados nesse estudo, a maioria apresentou cura para a doença, o que indicaria a conclusão do tratamento. Vários fatores influenciam o indivíduo para o abandono, como informações precárias sobre a doença, uso de entorpecentes lícitos ou ilícitos como álcool e tabagismo, crença na cura por meio mágico-religioso, nível socioeconômico baixo, intolerância às medicações de tratamento, diminuição dos sintomas ao início do tratamento, quantidade grande dos comprimidos, escolaridade baixa, entre outros, sendo necessária a reestruturação dos serviços de saúde dentro de uma abordagem que considere fatores psicossociais do indivíduo (SÁ AMM, et al., 2017). 
Vale ressaltar que a ficha de notificação preenchida pela equipe do ambulatório de tuberculose do município estudado e a posterior digitação desses dados no sistema TBWEB, apontou a necessidade de aperfeiçoamento no processo de preenchimento dos dados pela equipe de saúde, tendo em vista a incompletude ou não preenchimento de algumas informações, gerando viéses para o presente estudo. Nesse sentido, o trabalho se restringiu a uma população e cenário específicos de uma única localidade, comprometendo a generalização dos achados. Outra limitação encontra-se em relação aos dados secundários, não permitindo definir relações de causalidade entre determinadas variáveis.

Por fim, apesar das considerações supracitadas, os resultados encontrados cumprem o papel de traçar um panorama dos aspectos clínicos e epidemiológicos dos indivíduos com TB no cenário de estudo, tendo em vista que são fundamentados em bancos de dados fornecidos pela Secretaria Municipal de Saúde. A análise apresentada nesse estudo poderá auxiliar como substrato para implementação de medidas preventivas para a mudança do cenário de saúde das pessoas portadores com tuberculose e mitigar o impacto naquelas populações que são susceptíveis.

\section{CONCLUSÃO}

O perfil epidemiológico das pessoas diagnosticadas e notificadas com tuberculose no município foram homens com média de idade de 41 anos, com baixa escolaridade e que tinham como agravante da doença hábitos de vida como o uso de tabaco, álcool e outras drogas. Salienta-se que os registros dos casos encontrados de tuberculose nesse município no período observado, mostra algumas dissonâncias, havendo a necessidade de maior atenção no registro dos mesmos por parte dos profissionais de saúde, visto que a subnotificação implica na dificuldade de compor ações efetivas em saúde que contribuam para a produção de respostas para o enfrentamento da doença. Os resultados sugeriram a necessidade de novos estudos que explorem as estratégias de controle da doença na atenção primária, observando formas de fortalecimento.

\section{AGRADECIMENTOS E FINANCIAMENTO}

Agradecemos o Comitê Interno do Programa Institucional de Bolsas de Iniciação Científica do Centro Universitário Municipal de Franca (Uni-FACEF), que possibilitou a realização do presente estudo e aos membros da Secretaria de Saúde do município em disponibilizar seus equipamentos para coleta dos dados.

\section{REFERÊNCIAS}

1. BASTOS SH, et al. Coinfecção tuberculose/HIV: perfil sociodemográfico e saúde de usuários de um centro especializado. Acta paul. enferm., 2020; 33: 1-7: eAPE20190051.

2. CAMPOY LT, et al. Qualidade e gestão da atenção à coinfecção tuberculose e HIV no estado de São Paulo. Texto Contexto Enferm [Internet]. 2019, 28: 1-16: e20180166.

3. CAVALIN RF. Coinfecção TB-HIV: análise espacial e temporal no município de São Paulo. Dissertação (Mestrado em Ciências) - Faculdade de Saúde Pública da Universidade de São Paulo. São Paulo, 2018; 129 p.

4. COSTA AFA, et al. Competências profissionais de promoção da saúde no atendimento a pacientes com tuberculose. Rev. Bras. Enferm., Brasília, 2020; 73 (2): 1-7: e20180943

5. DANTAS DNA, et al. Fatores associados ao atraso na procura por atendimento pelo doente de tuberculose. Rev Bras Enferm [Internet], 2018; 71(supl1):691-6.

6. FREITAS WMTM, et al. Perfil clínico-epidemiológico de pacientes portadores de tuberculose atendidos em uma unidade municipal de saúde de Belém, Estado do Pará, Brasil. Rev Pan-Amaz Saude 2016; 7(2):45-50.

7. GIERGOWICZ FB, MAHMUD IC. A população privada de liberdade e a tuberculose: perfil epidemiológico em Porto Alegre/RS. Scientia Medica Porto Alegre, 2020; 30:1-8.

8. INSTITUTO BRASILEIRO DE GEOGRAFIA E ESTATÍSTICA (IBGE). Panorama de Franca-SP. 2020. Disponível em: https://cidades.ibge.gov.br/brasil/sp/franca/panorama. Acesso em: 29 de janeiro de 2021

9. JUNGES JR, et al. Tratamento Diretamente Observado da tuberculose: análise crítica da descentralização. Interface (Botucatu), 2020; 24: 1-13: e190160.

10. LEITE RC. Intervalo do tempo decorrido entre a investigação diagnóstica laboratorial e o início do tratamento em casos de tuberculose pulmonar em um distrito da atenção primária de saúde em Recife - PE. Dissertação (Mestrado Profissional em Epidemiologia e Controle da Tuberculose) - Centro de Pesquisas Aggeu Magalhães e Escola Nacional de Saúde Pública Sérgio Arouca, Fundação Oswaldo Cruz, Recife, 2016; 87 p. 
11. MALACARNE J, et al. Desempenho de testes para o diagnóstico de tuberculose pulmonar em populações indígenas no Brasil: a contribuição do Teste Rápido Molecular. J. bras. Pneumol., 2019 45(2): 1-7.

12. MINISTÉRIO DA SAÚDE. Secretaria de Vigilância em Saúde. Boletim Epidemiológico de Tuberculose. $1^{\underline{a}}$ ed. 2021. Disponível em: https://www.gov.br/saude/pt-br/media/pdf/2021/marco/24/boletim-tuberculose-2021_24.03. Acesso em: 14 de agosto de 2021.

13. MINISTÉRIO DA SAÚDE. Secretaria de Vigilância em Saúde. Departamento de Vigilância Epidemiológica. Manual de Recomendações para o Controle da Tuberculose no Brasil. 2 $2^{\underline{a}}$ ed. 2019 . Disponível em: https://bvsms.saude.gov.br/bvs/publicacoes/manual_recomendacoes_controle_tuberculose_brasil_2_ed.pdf. Acesso em: 14 de agosto de 2021.

14. MINISTÉRIO DA SAÚDE. Secretaria de Vigilância em Saúde. Departamento de Vigilância das Doenças Transmissíveis. Panorama da tuberculose no Brasil: diagnóstico situacional a partir de indicadores epidemiológicos e operacionais. $11^{\underline{a}} \quad 2018 . \quad$ Disponível https://bvsms.saude.gov.br/bvs/publicacoes/tuberculose_brasil_indicadores_epidemiologicos_operacionais.pdf. Acesso em: 8 de fevereiro de 2021.

15. MINISTÉRIO DA SAÚDE. Secretaria de Vigilância em Saúde. Departamento de Vigilância das Doenças Transmissíveis. Brasil Livre da Tuberculose: Plano Nacional pelo Fim da Tuberculose como Problema de Saúde $\begin{array}{llll}\text { Pública. } & 1 \underline{a} & \text { ed., } & \text { Disponível em: }\end{array}$ https://bvsms.saude.gov.br/bvs/publicacoes/brasil_livre_tuberculose_plano_nacional.pdf. Acesso em: 14 de agosto de 2021.

16. MINISTÉRIO DA SAÚDE. Secretaria de Vigilância em Saúde. Departamento de Doenças de Condições Crônicas e Infecções Sexualmente Transmissíveis. Boletim epidemiológico de HIV e Aids. 2020. Disponível em: file://C:/Users/Usu\%C3\%A1rio/Downloads/boletim_hiv_aids_2020_com_marcas\%20(2).pdf. Acesso em: 14 de agosto de 2021.

17. PINTO PFPS, et al. Perfil epidemiológico da tuberculose no município de São Paulo de 2006 a 2013. Rev. Bras. Epidemiol, jul-set, 2017; 20(3): 549-557

18. PONCE MAZ, et al. Atraso do diagnóstico da tuberculose em adultos em um município paulista em 2009: estudo transversal. Epidemiol. Serv. Saude, Brasília, jul-set 2016; 25(3): 553-562.

19. RANZANI OT, et al. Quem são os pacientes com tuberculose diagnosticados no pronto-socorro? Uma análise dos desfechos do tratamento no Estado de São Paulo, Brasil J Bras Pneumol. 2018; 44(2): 125-133.

20. RÊGO RT, et al. Perfil epidemiológico da tuberculose em Juiz de Fora, município prioritário no estado de Minas Gerais. HU Revista, Juiz de Fora, 2018; 44(3): 343-350.

21. SANTOS JUNIOR CJ, et al. Aspectos clínicos e epidemiológicos da tuberculose em pacientes com HIV/aids. Medicina (Ribeirão Preto. Online) 2019; 52(3): 231-8

22. SÁ AMM, et al. Causas de abandono do tratamento entre portadores de tuberculose. Rev Soc Bras Clin Med. 2017; 15(3): 155-60

23. SECRETARIA DE SAÚDE. Prefeitura digital. Franca, 2021. Disponível em: https://www.franca.sp.gov.br/administracao-municipal/administracao-direta/saude/apresentacao-saude. Acesso: 30 de janeiro de 2021

24. SILVA LT, et al. Perfil epidemiológico da tuberculose no serviço de referência do estado de Rondônia. Rev. Epidemiol. Controle Infecç. Santa Cruz do Sul, 2019; 9(1): 48-54.

25. SILVA TO, et al. População em situação de rua no Brasil: estudo descritivo sobre o perfil sociodemográfico e da morbidade por tuberculose, 2014-2019. Epidemiol. Serv. Saude, Brasília, 2021; 30(1): 1-12: e2020566.

26. TAVARES CM, et al. Tendência e caracterização epidemiológica da tuberculose em Alagoas, 2007-2016. Cad. Saúde Colet., 2020; 28(1): 107-115.

27. THOMÉ HR et al. Características clínicas, epidemiológicas e georreferenciamento da tuberculose em um centro de referência do oeste do Paraná. R. Saúde Públ. Paraná. 2020; 3(1): 86-96

28. TOMBERG JO, et al. Comportamento de busca por serviços de saúde para a detecção da tuberculose. Rev. Enferm. UFSM - REUFSM, 2020; 10 (52):1-18

29. TONIN E. Tabagismo em pessoas com tuberculose: características sociodemográficas, clínicas, diagnósticas e de acompanhamento. Dissertação (Programa de pós-graduação em Saúde Pública em região de fronteira - Mestrado) Universidade Estadual do Oeste do Paraná, Foz do Iguaçu, 2021. 45 p.

30. WORLD HEALTH ORGANIZATION (WHO). Global tuberculosis report 2020. Geneva: World Health Organization; 2020. Disponível em: https://apps.who.int/iris/bitstream/handle/10665/336069/9789240013131-eng.pdf. Acessado em: 19 de outubro de 2021. 